\title{
Narrative Substrates: Reifying and Managing Emergent Narratives in Persistent Game Worlds
}

\author{
Viktor Gustafsson \\ gustafsson@lri.fr \\ Université Paris-Saclay, CNRS, Inria \\ Orsay, France
}

\author{
Benjamin Holme \\ ben@holme.be \\ Benjamin Holme Design \\ Stockholm, Sweden
}

\author{
Wendy E. Mackay \\ mackay@lri.fr \\ Université Paris-Saclay, CNRS, Inria \\ Orsay, France
}

\begin{abstract}
Players in modern Massively Multiplayer Online Role-Playing Games progress through ambitiously designed narratives, but have no real influence on the game, since only their characters' data, not the game environment, persists. Although earlier games supported player influence by persisting changes in the world, they relied on players' capacity to form their own stories and lacked guidance for character progression. We explore how persistence and narrative emergence let us build upon players' influence rather than restrict it. We ran four studies and found that players highly value first-time and unique events, and often externalize their experiences to the Web to collaborate and socialize, but unintentionally also disrupt some aspects of in-game play. We introduce Narrative Substrates, a theoretical framework for designing game architectures that represent, manage, and persist traces of player activity as unique, interactive content. To illustrate and test the theory, we developed the game We Ride and deployed it as a two-phase technology probe over one year. We identify key benefits and challenges of our approach, and argue that reification of emergent narratives offers new design opportunities for creating truly interactive games.
\end{abstract}

\section{CCS CONCEPTS}

- Human-centered computing $\rightarrow$ Human computer interaction (HCI); • Information systems $\rightarrow$ Massively multiplayer online games.

\section{KEYWORDS}

Emergent narratives, Multiplayer game worlds, Persistence

\section{INTRODUCTION}

We are interested in the role of persistence in Massively Multiplayer Online Role-Playing Games (MMORPGs). In the real world, persistence is an implicit property of the environment, where objects grow, change and continue over time. Virtual worlds are persistent if they evolve, at least to some degree, and continue to exist even with no further interaction [4]. Players interact with and affect such games differently, depending upon how persistence is designed.

World of Warcraft's (WoW) [10] success made it the industry standard for designing such games [58, 59], although at the expense of players' influence on the world [53]. Earlier games, e.g., Multi-User Dungeons (MUDs) and Ultima Online (UO) [2, 45], let

FDG '20, September 15-18, 2020, Bugibba, Malta (C) 2020 Association for Computing Machinery.

This is the author's version of the work. It is posted here for your personal use. Not for redistribution. The definitive Version of Record was published in International Conference on the Foundations of Digital Games (FDG '20), September 15-18, 2020, Bugibba, Malta, https://doi.org/10.1145/3402942.3403015. players add and move objects in a shared, open world environment. The resulting changes persist in the world, so players collectively contribute to an evolving, unofficial narrative that emerges from their actions but lack a compelling overall story with character progression.

By contrast, most current games reward players for following a pre-written narrative. Game designers guide character progression, and unlike earlier systems, players cannot add objects nor leave traces that permanently influence other players' experiences. Since only character data persists [35], when players log out, it is as if they never existed [53] and had no impact on the game.

Game designers take full responsibility for entertaining players by adding new content-players in non-persistent worlds cannot contribute, nor can the environment grow by itself. Ambitious players who consume all available content must thus wait for designers to add more. Unfortunately, developing content takes far more time than consuming it, and game designers struggle to meet the demands of motivated players. Debeauvais et al. [17] report that designers can no longer fully satisfy these WoW players' needs: Even when they plan strategically and distribute patches with new content over long periods of time, they lose players.

We are interested in how we can address this challenge by leveraging persistence i.e. reification, which turns user actions into first class objects that act as tools [6], to capture traces of players' activities to produce novel content. What if games could persist traces of players' stories and reify them into interactive gameplay elements? What if players' past actions could generate 'legendary' artifacts with additional powers? What if locations could adapt themselves, according to past events, enabling prophecies to come true? Players would become real legends, remembered and influential, and contribute to emergent narratives that enhance rather than restrict players' influence. To achieve this vision, we first need to better understand how persistence affects game play, and the challenges it raises for designers, e.g., how can designers select what is meaningful to persist without being flooded with data? How can they predict the long-term consequences of persistence, e.g., if a player or item becomes too powerful?

This paper explores how to increase players' influence through persistence, and how to selectively choose and reuse narrative elements that emerge from their actions. We first review related work, then describe the results of four studies and how they informed the design concept we call Narrative Substrates. We next describe the design and implementation of We Ride, a working MMORPG, and its year-long deployment as a technology probe [31]. We conclude with a discussion of the potential benefits and challenges of Narrative Substrates, with directions for future work. 


\section{RELATED WORK}

We first examine how early research defines 'persistence' with respect to players' ability to change the game world. We also examine the narrative potential offered by game worlds, including how two games incorporate emergent game mechanics and persistence in Human-Computer Interaction research.

\subsection{Persistence in Game Design}

Persistent features in early MMORPGs were introduced by virtual game world pioneers Bartle and Koster. Bartle co-founded the first Multi-User Dungeon (MUD) in 1978 [4] and has been exploring and teaching about the infrastructure of virtual worlds ever since. Koster led the design of Ultima Online and Star Wars Galaxies, expanding and transforming their concepts from MUDs into graphical computing. He describes the typical process of persistence in two steps [35]: First, designers set up definitions and game rules in a static 'read-only' template database, for example, defining a new character's starting state. Next, players play according to these rules, causing them to accumulate items and evolve skills that are communicated to a server and saved to a dynamic runtime database. The stored data persists as the server continuously recommunicates data to the client. Although still widely used, this solution forces reliance on a single server, or splitting the world into different zones, each with a server. Moving into a different zone causes a screen reload, which disturbs the illusion of a large, seamless world.

Koster [36] also discusses how changes users make to the world can produce consequences that persist in the world. He argues that persistence is best understood as a spectrum, with a relationship between degrees of persistence and players' ability to change the world. He highlights a key challenge for designers: How to choose the 'granularity and sophistication' of what persists? [34]. For example, persisting positions of blades of grass requires high granularity, but may have no relevant effect on the player's experience.

Tychsen and Hitchens' [53] narrative analysis explores how MMORPGs rely on traditional storytelling techniques to manipulate time, but struggle to provide players with meaningful consequences. Their model for time-based consequences compares internal consequences, affecting the character itself, with external consequences, affecting the environment and other characters, in terms of temporal duration and the magnitude-non-permanent, limited permanence or permanent-of these consequences. They argue that designers can bypass some of these issues and support a wider range of storytelling techniques with instances-confined areas that replicate themselves for the players or groups that enter them. This entitles players to that space's content and separates the consequences of their actions from the rest of the game world. We seek long-term solutions where players influence each other through shared stories, not just rich, but independent activities.

\subsection{Narrative Potential and Emergence}

Koster [36] describes how game worlds can offer persistent features to players-letting players produce features that persist in the game world-but not the reverse. Although few researchers study persistence as a first class object, several explore the narrative potential of persistence in game worlds.
Fencott defines narrative potential as 'the accumulation of meaningful experience as a result of agency [that] allows users to construct their own appropriate narratives' [22]. Eladhari and Lindley [20] go further, describing it as the possible narrative outcome of a designed environment and players' interaction within it, which enables possible 'histories of play' to emerge as stories are retold. Ryan [49] defines emergent narrative as a phenomenon produced by a system that "simulates a story world out of which narrative may emerge from the happenstance of character activity in that world". His dissertation explores the differences between successful and failing storytelling games, and suggests that it relies on how the world's content supports players' ability to curate their own stories. He argues that the thrill of emergent narratives is closer to non-fiction than fiction, where emergent events really happen, unlike pre-written narratives where every consequence is a calculated part of the design.

Madden and Logan [42] describe a framework for generating a collaborative narrative where witness-narrator-agents foster a sense of community and motivate players. Although these agents function as Non-Playing Characters (NPC), they also report and present events involving players, recognized from a dedicated narrative ontology. This use of agents lets players participate in and help control narrative generation, rather than having the game do it for them automatically. Players are also aware that they are observed, and can explicitly direct the agent's attention to certain events.

Pita et al. [46] argue that MMORPGs lack unique quest experiences, and explore how to generate adaptive narratives based on player input. They present the TRUE STORY framework for reusing history and referencing relationships among objects to continuously generate unique quests. They developed a set of constraints that help designers define how they want to generate content, e.g. memories, attributes, actions, layers and proximity. They implemented the framework in an offline, text-based persistent world to show how to reuse past player activity to generate new quests, but have not actually tested it. We would like to go beyond the scope of generating new quests for players, and create solutions that support the reification of player histories across the entire architecture of graphically rich game online worlds.

\subsection{Persistent Games: Two Examples}

We review two games that are often recognized for their unique features by the gaming community. Neither provide fully persistent game worlds, but they introduce relevant concepts related to persisting and reusing player history.

Bloodborne [25] is an Action Role-Playing Game (ARPG) where players not only try to progress in the game, but can also leave each other notes that persist and are distributed to other players' instances of the game. These notes function as warnings or clues to upcoming events. The content of each note is restricted to a predefined, one-sentence template, to which a player can add phrases and selected words, or even a gesture to be performed by a ghost avatar [57]. Since all play in identical worlds, the game simply sends a location's coordinates over the network, and implements it with the other player client. Although Bloodborne is not a MMORPG, it still offers an interesting example of persistence, with an underlying semantic structure. Players have a tool that lets them affect other 
players' gameplay, and the mechanics of this form of persistence could be implemented for a large audience.

Middle-earth: Shadow of War [44] is an open-world ARPG set in the Lord of the Rings universe and features what they call the Nemesis System. It is designed to let NPCs evolve based on player's actions and re-appear in different encounters, creating '...unique personal stories with every enemy and follower...' [24]. When players encounter high-ranking enemies in Sauron's army, their actions influence how enemies develop and change, affecting their personality, traits and rivalries. Enemies can develop various characteristics, such as unique names, appearance, ranking within the army and even special fighting styles [32]. If players are defeated, the enemy grows stronger and becomes the player's 'nemesis', able to hunt down the player anywhere in the world, and build on previous encounters. The implementation of this system proved challenging to developers, requiring them to work with multiple departments simultaneously and think 'three to four steps ahead all the time' [16]. The game is not multiplayer, but shows how another kind of system, focused on an environment that responds to and evolves from players' actions, could generate novel storytelling features [38].

\subsection{Persistence in HCI Research}

Researchers in Human-Computer Interaction (HCI) have explored persistence as a means of reusing past user activity to navigate digital interfaces. For example, History-Enriched Digital Objects (HEDO), based on Distributed Cognition [30], highlights the potential benefits of persisting and representing activity traces, especially for ethnographic studies. Hill et al. [29] apply this concept to customized scrollbars that capture the idea of computational wear. Users see a representation of their interaction history, including previous edits and the parts of a document they read, which reveals their past actions and helps collaborate more effectively.

Schütte [51] presents PatinaMap, a web-based image map for capturing history of use, and demonstrate the benefits of various methods of representing wear. For example, they generate noises depending upon a location's popularity; create visual filters that actively adapt to meta-data; and support annotations of synchronous interaction, e.g. by displaying icons of user's mouse-clicks to create awareness of each others' presence.

Wexelblat and Maes [56] explore how such functionality can aid navigation in information-rich spaces. They describe Footprints, a set of tools for visualizing users' web-browsing histories based on user-frequency data, location-visit maps, annotations, and graphs of navigation paths. A year-long longitudinal study of Footprints showed that the same amount of work can be accomplished with significantly less effort. Dourish and Chalmers [18, 19] introduce the concept of Social navigation, where persisted traces mediate between past and present user navigation of a location. They suggest that users can indirectly help each other navigate by leaving traces of their activity, thus potentially reshaping the space's structure without direct involvement by designers.

\section{RESEARCH APPROACH}

Although previous research offers insights and perspectives on persistence with respect to narrative theory and games, it does not offer concrete guidelines for designing massive online systems where players' activity directly influences the game world. The latter requires a deeper understanding of players' current experiences, and raises a number of challenging methodological issues.

Different methods offer different trade-offs: Interviews provide rich detail about play activity, especially if the interviewer probes for problematic or surprising occurrences. Unfortunately, interviews are time consuming, rely on the person's memory and are necessarily limited in scope.

By contrast, questionnaires are easy to deploy and reach a larger audience, but usually focus on demographic and opinion data, rather than useful insights about the user experience. We can also learn general characteristics of players through quantitative analysis of open game databases [15].

Eladhari [21] proposes another approach, where examining the stories players retell offers valuable insights into their experiences, since they took the time to write and share them. Kreminski et al. [37] evaluate AI-based games by gathering players' stories from Red$d i t^{1}$, and interviewing their authors. They calculated the frequency of various game elements, and drew conclusions about different aspects of the game.

We use a triangulation approach [41] to balance the trade-offs across methods by gathering multiple types of empirical data, and building on and extending theory to generate novel designs. We ran four studies, using interviews, story questionnaires and Reddit posts, to understand the current role of persistence in games.

\subsection{Study One: Interviews}

To gather stories related to a particular game artifact, we used a variation of the critical incident technique [23] and conducted critical object interviews $[39,40]$ with five men, ages 21 to 34 . Online interviews, using Discord, lasted about 60 minutes. We asked participants to recall a recent MMORPG session and describe a meaningful object they owned in the game. We probed for contextual information about how they obtained it and why it was meaningful. If they had an active account in the game, we asked them to share their screen and show us the relevant object. The results highlighted the importance of gathering many short specific stories, rather than a few very long, detailed ones, when trying to understand the role that persistent objects play in enhancing the user experience.

\subsection{Study Two: Story Questionnaires}

We learned from the interviews and developed story questionnaires [28], which combine the critical-object interview technique with a shortanswer questionnaire. We prepared sample stories, derived from the interviews, to encourage participants to remember, reflect and respond back with similar stories.

Versions one and two of the questionnaire asked for a story about a meaningful or memorable game experience, then presented the sample stories as potential triggers. We asked follow-up questions after each story, to determine the level of impact on the game, and if they shared the story with someone else. Version three removed the initial question and only presented sample stories, with new follow-up questions that focused directly on persistence. Specifically, we asked if any traces remained in the world from the story;

\footnotetext{
${ }^{1}$ Reddit hosts user-moderated communities, where users post, comment, and vote "up" or "down" each others' contributions.
} 
the importance of those traces for enjoying the game; if the participant would like others to find those traces; and if so, why and how? Each participant responded to only one version of the questionnaire. We recruited participants from both gaming specific online spaces, e.g., MMO-champion.com and Discord, to more general subgroups on larger social networks, e.g., Reddit, Facebook and Twitter. We collected 65 stories from 40 respondents.

\subsection{Study Three: Published Reddit Post}

We created a Reddit post to gather examples of games that inspire creation of long-lasting narratives. We were especially interested in narrative persistence, including any barriers to implementation. We published a post [48] on the truegaming ${ }^{2}$ sub-community, which facilitates elaborate discussion around games. Under the title: "What games foster players to create their own legends?' we asked: “Are there any MMOS out there that allow players to create and share their own epic legends based on in-game experiences?" We said we knew of persistent games such as EVE Online [13] and UO, and asked specifically for generative features, that capture and let narratives evolve as the game proceeds. We also probed users for follow-up comments if we thought it might lead to more information. Respondents could comment and vote (once) on the original post or on each other's comments. We collected 71 posts from 47 respondents.

\subsection{Study Four: Independent Reddit Post}

We extracted and analyzed comments from an independently created Reddit post [47], entitled: "Gamers of Reddit, what gaming experience will you never forget and why?" At the time of data extraction on 12 June, 2019, the post was $89 \%$ upvoted and had approximately 15000 comments. We used PRAW ${ }^{3}$ to create a script that extracted comments and parsed them for keywords related to MMORPGs, including full titles, abbreviations, and expansion packs. We collected 293 posts from 288 respondents.

\subsection{Data Collection}

In addition to voice recordings from the interviews, we collected a total of 434 text-based stories from 380 respondents over the four studies (see Table 1).

\begin{tabular}{|c|c|c|c|}
\hline Study & Method & Stories & Respondents \\
\hline $\mathbf{1}$ & Interview & 5 & 5 \\
\hline $\mathbf{2}$ & Story Questionnaire & 65 & 40 \\
\hline $\mathbf{3}$ & Published Reddit Post & $71^{*}$ & 47 \\
\hline $\mathbf{4}$ & Independent Reddit Post & 293 & 288 \\
\hline & & $\mathbf{4 3 4}$ & $\mathbf{3 8 0}$ \\
\cline { 3 - 4 } & &
\end{tabular}

Table 1. Total stories and respondents per study.

${ }^{*}$ Total comments selected that relate to persistence in game play.

\subsection{Data Analysis}

We analyzed the stories using Thematic Analysis [11], with a mix of bottom-up (inductive) and top-down (deductive) approaches. First,

\footnotetext{
${ }^{2}$ https://www.reddit.com/r/truegaming/

${ }^{3}$ https://praw.readthedocs.io/en/latest/
}

we read through each story and developed an initial coding for all words and concepts we deemed relevant to 'meaningful experiences' and 'persistence'. Then, we filtered the codes by eliminating synonyms and grouping closely related concepts to generate an initial set of themes. We iterated this process several times while counting codes, themes and their prevalence in a spreadsheet. We also analyzed the follow-up questions from study two, then summarized and cross-referenced the answers in relation to the stories.

\section{RESULTS AND DISCUSSION}

We present our findings with their corresponding prevalence in the dataset and discuss how they relate to persistence and player influence. We conclude with four design implications which are then developed into the theory of Narrative Substrates.

We ran a bottom-up analysis of studies $1 \& 2$ and selected two key themes, uniqueness and meta persistence, which guided our top-down analysis of studies $3 \& 4$. All four studies indicated that the lack of world persistence lowers player's sense of uniqueness and that they leverage persistent features of the Web to such extent that it also disrupts game play. We also found that, in relation to uniqueness, players' stories are significantly influenced by firsttime experiences and that persistence affects the rate at which game worlds remain relevant and interesting to players.

\subsection{Unique and First-Time Experiences}

'MMORPG' is a broad term that includes two key subgenres. Most modern games, e.g., WoW, are 'theme parks'-static environments designed for plot-driven events linked to character progression [3]. Older games, e.g., UO, are 'sandboxes'-malleable environments that promote gameplay-driven events by giving players tools and freedom to act as they wish $[3,36]$. Hybrids combine the two, with some plot and a somewhat-persistent environment.

The most upvoted MMORPG-related comment in study three $\left(S_{3}\right)$ highlights the difference between theme parks and sandboxes:
"Most MMORPGs are just theme parks. If you remove competitive play and grinding from the games, they'd work better as a simple online co-op RPG. The illusion of a living world is usually paper-thin. Sandboxes [...] tend to favor freedom over story, and it shows. Any sto- ries and legends coming out of these games are written entirely externally." (Participant 8 , Study 3) $\left(P_{8}, S_{3}\right)$.

The comment illustrates a key gap between MMORPG genres: Each has advantages and disadvantages, with different relationships to persistence. We use this distinction to structure our discussion of how players' unique experiences are affected by different design choices involving persistence.

Uniqueness is Difficult to Control \& Represent. Players want unique content, but designers lack viable ways to offer it. In study $4\left(S_{4}\right)$, we saw that players are most likely to remember events from the first time they experienced them. This was especially common in theme parks where $61 \%$ of all stories were characterized by the thrill of achievement and discovery.

Memories of first-time achievements are far more common in theme parks (88\%) than in hybrid games $(9 \%)$ and sandboxes $(3 \%)$. They signify predefined feats within the game or goals created by 
the players themselves. Examples include defeating bosses, reaching the final level, retrieving special items and defeating rival players.

One benefit of pre-written narratives is their ability to communicate and prepare which achievements are available within the game. Theme parks offer separated modes of Player-versus-Player (PvP) and Player-versus-Environment (PvE), each with their own systems of rewards that nudge players toward pre-defined, discernible goals: "Getting Wrecking ball achievement in WoW battlegrounds more than 10 years ago. The achievement requires killing 20 or more players from opponent team without dying once. I finished the match with 30:0 kills/deaths." $\left(P_{108}, S_{4}\right)$.

In hybrids and sandboxes, PvP and PvE often mix, and are spontaneous and consequential, stirring up conflict among players that lead to surprising outcomes. However, such moments are more difficult for designers to define and formalize as achievable goals, although they may represent valuable moments for players.

Game designers offer players greater agency at the cost of decreased control over the consequences, making it harder to predict which achievements players will enjoy and how to represent and reuse them. Theme parks actively prevent players from influencing the world, to avoid exposing players to uncontrolled conditions and complicating their ability to predict outcomes and manage player expectations. Instead, they use narrative to give everyone the same illusion that they can influence the world.

Non-Persistent Worlds 'Shrink' With Time. We found that many players ( $11 \%$ of all posts, $S_{4}$ ) enjoy discovering vast worlds together, especially for the very first time, e.g., when the technology was new to support thousands of players simultaneously: "First time I played World of Warcraft right before Burning Crusade came out. The world was huge and filled with so many people" $\left(P_{248}, S_{4}\right)$.

Players also remember their journeys traversing different locations of the world: "Friend showed me how to make a NE and run to Darnassus, portal to Rut'theran, boat to Darkshore - Auberdine, boat to Wetlands - Menethil Harbour, run to Loch Modan, then run to Iron Forge and get the tram to Stormwind then run to the keep. That was a wild few hours." $\left(P_{280}, S_{4}\right)$. Such journeys are typical early in the game, when players want to play with friends at another starting location or just prefer a different environment, but want to be a special type of character. In theme parks however, once players reach the elder game; what comes after they have finished leveling; e.g., raiding, PvP, daily quests or 'instance grinding' [5], exploration declines and players can only progress by repeating old content.

Since the world neither persists nor changes when players interact, nothing new or interesting can emerge. Players' motives to explore and reside in areas decline with time. They do not want to spend time travelling over areas that they already know. Instead, they simply teleport to instantiated locations that benefit their progress, shrinking the 'open-world' aspect of the game.

Control Leads to Repetitive Content. If events are easy and repetitive, they become ordinary and not unique. Yet making difficult, custom-made events requires too many development resources for too few players, making it hard to manage players' expectations. Two respondents mentioned modern MMORPGs, Guild Wars 2 [1] and Final Fantasy XIV [52], where events are designed to shape the world. However, they occur too often and instead break the illusion when players realize they are not unique: "Having them be on a loop and always happen really does diminish how fun they are. Instead of being a cool event that changes the world, they're just business as usual. [I prefer] events like that be much less frequent..." $\left(P_{10}, S_{3}\right)$.

Players learn that they are the hero of the narrative and expect that other players have the same opportunities if they spend the same amount of time. "The problem with MMORPGs is that you have to maintain a status quo. You can't make the player experience unequal, unless you're hardcore like EVE Online...What do people whine about the most when it comes to the granddaddy MMO of them all, World of Warcraft? Balance patches...” $\left(P_{44}, S_{3}\right)$.

Sandboxes offer unique content that emerges from players' actions, but lack ways to represent them and are considered hardcore because they offer 'unequal' player experiences. By contrast, theme park designers balance systems and produce controlled new content, which lets every player achieve hero status in the official narrative, even if the illusion often breaks down.

\subsection{Meta Persistence}

We found that $30 \%$ of all stories in $S_{4}$ involve accounts of the real world and that persistence plays a particular role in 'meta gaming', i.e. what players consider play between the game and the real world $[12,50]$. Players consistently externalize their stories and observations to the Web. This 'meta persistence' lets players share, socialize and collaborate around games. We also found that the magnitude of players' voluntary efforts to contribute often disturbs play in theme parks and hybrids, although less so in sandboxes, since GMs can curate 'meta-persisted' stories into new content without conflicting a set official narrative.

Knowing Too Much Spoils The Fun. The open relationship between a game world and its surrounding culture can have a negative impact on gameplay. Players collaborate to create rich, web-based information resources and tools to help them optimize and finish gameplay tasks. However, games are not always designed to sustain such efforts: "Meta gaming has fundamentally altered the reality of MMOs...all the details are online, mined, extracted, analyzed and published, usually before the content patch is even live..." $\left(P_{37}, S_{3}\right)$.

One participant in $S_{1}$ did not enjoy playing with these 'knowledge hubs': being aware that everyone was using them made him feel like he was the only one not playing with cheat codes $\left(P_{2}, S_{1}\right)$. $P_{45}, S_{3}$ argued that even if game legends emerge organically, players still mine data, optimize and write walk-through-guides for such processes. However, we also found that only $17 \%$ of all meta gaming related to retelling stories featured sandboxes, but was much more common in theme parks (34\%) and hybrids (40\%).

Meta persistence affects sandboxes less since the gameplay is open-ended and less structured. Pre-written and linear narratives are easier for players to reconstruct and optimize, hence game designers who already struggle to supply enough content, compete against large communities who specialize in consuming it.

Players Retelling Stories Outside The Game. Players not only create knowledge hubs and tools outside games, but also share stories of their in-game experiences. One respondent suggested that strategy games most easily facilitate player-created legends: "The more popular forms of Let's Play before YouTube really took off was the [...] After-Action Report, [...] basically a piece of fiction incorporating 
screenshots of gameplay, which act as the skeleton of a plot. One of the biggest forums for the Total War games even had a whole sub forum dedicated to these" $\left(P_{33}, S_{3}\right)$.

Gameplay generates stories, but if the game world is not persistent, these cannot be shared or built upon within the game. In Study $S_{2}, 71 \%$ of respondents could not find any traces of the stories they told, even though a majority wanted to. Instead, they captured playbased artifacts and retold their stories outside the game. By contrast, those who did find traces (29\%), appreciated them: "I really like the fact of leaving trace in the world. That reinforce[s] the idea of a world with a real time and background" $\left(P_{20}, S_{2}\right)$. They are also interested in how it works and how they could control such functionality: "I' $m$ not sure how a world could actually show traces of players individual stories in an MMO without cluttering up the world. How do we figure out which stories are worth making a permanent part of the world and which are not?...” $\left(P_{8}, S_{2}\right)$. This raises an interesting question about relevance with respect to players' stories. Clearly not all traces of players' stories should persist, since they could 'clutter' the world with potentially uninteresting details. This suggests that we need ways to detect and filter narratives that both fit the world and are of interest to other players.

In MMORPGs, theme parks cannot let player narratives affect overall gameplay, since they conflict with the promise of an equal player experience. By contrast, sandboxes do let players create their own narratives, but lack ways of representing them in the game. Although it affects the genres in different ways, both player communities externalize their stories to the web which lets them persist and grow rather than leaving traces inside the game.

Game Masters Curate Player Stories Into Content. We found that Game Masters (GMs) take on various roles, and actively 'morph' or contribute to players' experiences to fit within game world narratives. Tychsen et al. [54] suggest that MMORPG GMs work more with control, community support and runtime updates, whereas GMs in Table-Top and Live-Action Role Playing Games, curate a higher degree of narrative involvement.

Five respondents in Study $S_{3}$ describe the positive impact of GMs who interact with players in-game. In Elite Dangerous [26], GMs track what happens and report it back through in-game press releases, or take on the role of an actual character but with extended abilities. "People within the dev team [...] write in-game press releases based on actions within star systems or overarching events in the in-game political, system which is based around affiliated player actions." $\left(P_{14}, S_{3}\right)$. They highlight positive aspects of player-designer interaction and how the game allows each to generate visible and important consequences from their actions.

In The Matrix Online [43], one participant noted that GMs manage the main characters, who randomly appear to interact directly with players $\left(P_{32}, S_{3}\right)$. This is also common in UO: "The GMs would constantly interact with the community, putting on events with lore daily, always custom designed and fun to take part in" $\left(P_{38}, S_{3}\right)$. GMs can access player input both from runtime and externally persisted resources, which lets them continuously craft new unique events and represent players' actions, to curate a collaborative narrative out of artifacts of players' experiences.

\subsection{Summary \& Implications for Design}

These four studies indicate that players value unique experiences and would like to persist traces of their actions. Current MMORPGs lack the necessary infrastructure for representing players' actions and real influence, causing players to revert to online communities where they persist stories and share information. Based on the above results, we identify four key implications for the design of persistent game worlds.

1) Design Space For Managing Narratives: Designers must manage and understand data to maintain control over the game and ensure that traces remain relevant. This requires an infrastructure that persists and exposes elements of players' narratives where designers can detect and manipulate relationships among events.

2) Structured Persistence: Designers need a structure for persisted data that directs which types of narratives emerge. This requires predefining events that record basic information, e.g., time, location and characters, so designers can construct threads of traces based on when, where and how players interact.

3) Relationship Detection: Designers should take advantage of the fact that each player is unique, and so are the consequences of their actions during play. This requires a data structure that can be searched for similarities and relationships, and has the potential for generating unique new content.

4) Representation and Discoverability: Designers must know how to represent player narratives in meaningful ways. This requires a strategy for attracting players' attention and guiding them towards exploring their history in a way that suits each player's context.

\section{NARRATIVE SUBSTRATES}

Despite players' demonstrated desire for uniqueness and persistence, current MMORPG architectures make it difficult to incorporate and manage them. We argue that designers need an underlying design space to help balance the inherent trade-offs when deciding which and how traces of players' activities persist, with different levels of access that include game masters and even players.

We introduce Narrative Substrates: a theory for designing game infrastructures that support persistence, management and reuse of player narratives. Elements of players' past experiences are preserved and turned into first class objects that the player, other players, and game masters all can interact with. This offers a growing source of meaningful new content, with the goal of generating unique, but interconnected play experiences.

\subsection{Theoretical Background}

We are inspired by the theoretical principles of Reification, Polymorphism and Reuse [9] from Instrumental Interaction [6], where Reification turns user actions into first class objects that act as tools; polymorphism lets users manipulate multiple types of objects with a single tool; and reuse captures prior user input and system output and turns them into first class objects for subsequent interaction.

For example, StickyLines [14] reifies the alignment command into a persistent interactive object that users can control, manipulate and reuse to align and distribute graphical objects. This technique offers a simpler, yet more powerful tool with significantly enhanced performance. We use a similar strategy to reify traces of user activity into interactive narratives within a persistent game world. 
The concept of 'substrates' builds upon these principles, and has been explored in various contexts, including Polyphony [27], for music composition, and Webstrates [33] which creates an environment for 'shareable dynamic media'. We build on Beaudouin-Lafon's [8] definition of substrates as a:

"digital computational medium that holds digital information, possibly created by another substrate, applies constraints and transformations to it, reacts to changes in both the information and the substrate, and generates information consumable by other substrates. Substrates are extensible, composable with other substrates, and can be shared. They provide the fabric of the digital world."

For example, spreadsheets are substrates consisting of a flexible grid of cells that structure user input data; support different types of representation, e.g., text, numbers, graphs, images and equations; and define relationships across cells. These principles offer designers generative power [7] to create richer and more varied design spaces from which to develop innovative solutions. We combine them with related research on History-Enriched Digital Objects [30] and Social Navigation [18, 19], which highlight the benefits of reusing user-created traces.

\subsection{Structure}

Narrative Substrates persist and structure traces of interaction in digital environments. They let designers and content curators detect and manage relationships across individual narratives that can be composed into meaningful collective histories.

Story Events are the smallest elements in Narrative Substrates, defined as persistent snapshots of events that occur in play and carry information about actors, objects, location, time, and event type. Our studies highlighted the importance of event types, i.e. achievements, first-time experiences and unique encounters, to ensure meaning and relevance in captured narratives. Since any actor, object or location can persist story events, designers should consider which ones best suit the style of their game. The structure of story events ensures that coherent stories are formed regardless of how events persist, but the amount of available data may be low, e.g., if only weapons carry story events in a game focused on character interaction. This structure provides a starting point for individual or local narratives that is simple enough to grow large, without jeopardizing the designer's ability to discover and formalize new and global narratives.

Story Artifacts are actors or objects affiliated with a set of story events. e.g., if a player uses an axe to chop down the world's largest tree it can trigger a story event which is written to the axe, and makes it a story artifact. Designers and game masters may also synthesize relationships across separate story events and reify them into new, but historical content. For example, a GM who noticed that a previous player had helped Elves grow that tree could write a letter (story artifact) from them to the player, asking for revenge.

\subsection{Relationships}

Once data flows into the Narrative Substrates, designers and game masters gain access to a semantic interface where they can identify relationships among story events and manage possible new story

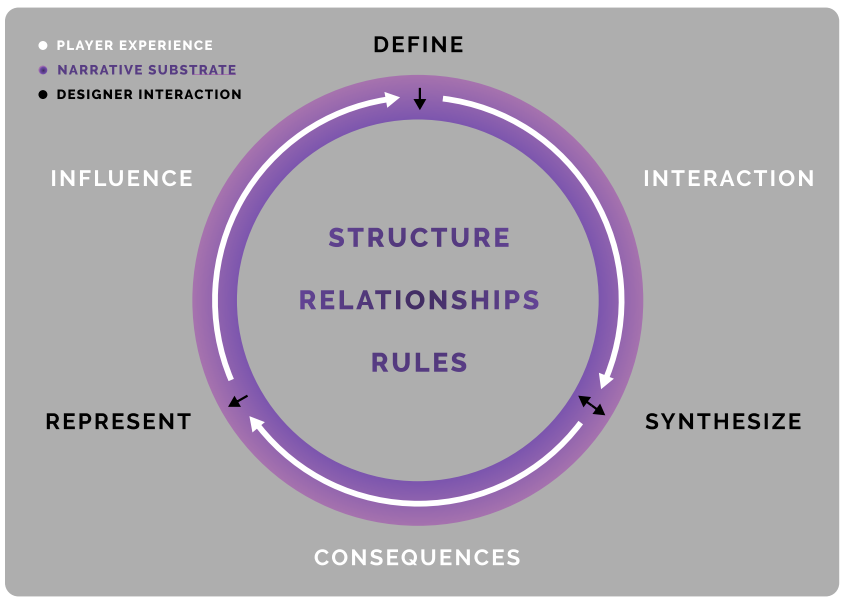

Figure 1. Narrative Substrates Process: Designers define the structure and synthesize relationships which, supported by rules, are reified into story artifacts that represent players' past actions and influence in the game world. Players' interaction produces consequences as raw data that is structured and persisted into story events.

strands. This is similar to an advanced form of the 'detective board' in films, where detectives track suspects, event timelines, and the relationships among them. Persisted story events can be filtered and displayed to help players discover new relationships and stories. Relationships can also be reified into links and chains of story events that can be grouped and manipulated together, equipping designers with powerful tools to conditionally spawn events or more easily manage prospective stories. Our study results suggest the following relationships are important, but additional, more sophisticated relationships should emerge as Narrative Substrates grow over time:

Co-occurrences create new relationships among story events that happen at the same time or place. For example, a character (actor) who visits a location wearing a sword (object) that had been found there earlier represents a historical connection or co-occurence.

Deviations mark unusual events or relationships as 'first-time' occurrences. For example, a legendary sword switching owner for the first time, or a player robbing the server's strongest player-guild.

Similarities organize similar or intersecting events into groups, which reduces clutter and detects linear or repetitive patterns in the game design. For example, players who often visit the same locations or items that share event type in a certain time period can be hidden to emphasize other events.

\subsection{Rules}

Narrative Substrates apply rules to story artifacts, so that players first discover, and then can interact with them in meaningful ways. We provide two basic categories of rules and encourage designers to add their own, as appropriate.

Interactivity: Story artifacts are inspectable, so players can learn why they exist; expandable, so the narrative continues to evolve as players interact with it; and shareable, so stories spread and players receive recognition and acknowledgment. 
Discoverability: Designers must consider how to reveal story artifacts so as to attract players' attention, invite interaction, and encourage players to explore their meaning further.

\section{WE RIDE: TECHNOLOGY PROBE}

We designed We Ride [55], a live, running early-stage 2.5D sandbox MMORPG, to explore how Narrative Substrates can persist player activity in an actual game world. We Ride offers a rich testbed for research, with a flexible, dynamic environment where we can test these concepts, and have players who are willing to experiment with it. We deployed it as a two-phase technology probe [31] that let us iterate through a series of design ideas with continuous player feedback, while preserving ecological validity.

\subsection{Deployment 1: Setup and First Impressions}

Deployment $D_{1}$ demonstrated how we can implement Narrative Substrates with objects as story artifacts and basic event types focused on first-time experiences. It also provided an opportunity for observing and capturing players' first impressions of the game.

Game World Description. Players share a large seamless world in a medieval fantasy setting, where they train to become a warrior, mage, ranger, or crafter. The world has no safe zones, and PvP is allowed everywhere, so if players die, they become ghosts. Everything they wear, plus inventory, stays on the ground for anyone to loot, until the player returns to the corpse after resurrection.

6.1.1 Participants. 114 players played during deployment $D_{1}$; four players continuously provided detailed feedback on Discord and responded to a questionnaire after the deployment.

6.1.2 Setup. We built We Ride with Unreal Engine 4 (UE4) and its blueprint visual scripting system. A server hosted in Sweden communicates with a MySQL database using PHP and the VaRest plugin ${ }^{4}$. We designed story artifacts as persistent objects that: record players' actions in the game; act as representations of historical records of personal player experiences in the context of the game world; and can be shared with other players, who can both expand its story as a new author and learn from it by reading its records. This illustrates how Narrative Substrates capture 'story events' that represent and form 'relationships', which in turn can be structured into 'links' and generate new 'story artifacts' based on 'rules'.

Players generate story artifacts by finding or crafting a highquality item, with a message displayed below their character explaining its historical relevance and ability to capture information. Players can inspect any story artifact to see its history (see Fig. 2). If a story artifact is a weapon, it counts how many enemies it has killed. One kill corresponds to 100 hit points, which rewards defeating more difficult enemies. Inspired by the Nemesis System, it also secretly tracks how many of each enemy race, e.g., 'orc' have been defeated, which signals the substrate to trigger conditionally spawning events based on that information. For example, if a weapon kills 100 orcs, it triggers and generates a new story event where orc assassins spawn and come to attack the player.

We defined the following story event types; owner change-a weapon is traded or looted, player kill-a player kills another player,

\footnotetext{
${ }^{4}$ https://github.com/ufna/VaRest
}

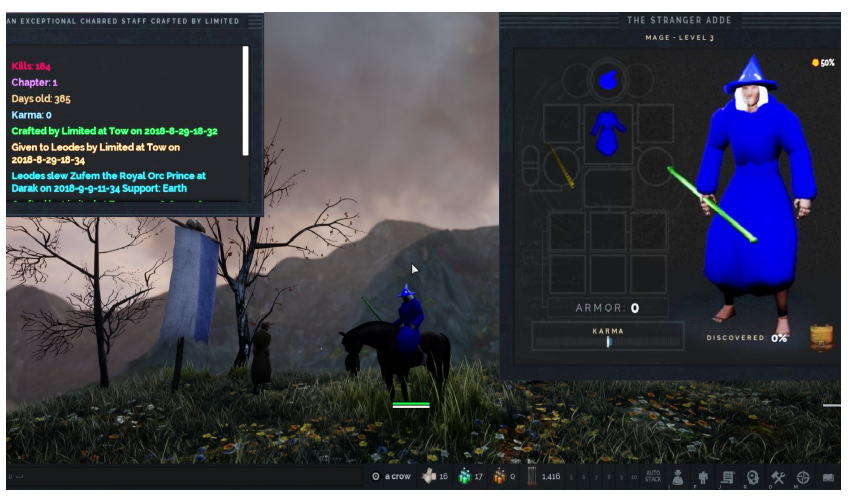

Figure 2. A We Ride player-character sits on a horse next to an honor banner, wielding a historical staff whose narrative lists: kills, chapter, age in real time, karma and story events in chronological order (upper left panel). Right panel shows the Character portrait.

and boss kill-player kills a boss. We also implemented a custom event type, so that GMs can organize scripted events that will persist.

Story artifacts evolve in levels or chapters where each new increment unlocks more functionality and visual characteristics. For example, a sword might change color and take on its owner's name, based on its number of kills, player kills and age. To encourage players to evolve weapons rather than just abandon them when they find a more powerful one, we built Pearl of Power, a rare item that transfers the damage modifier of any weapon to a story weapon.

Inspired by Madden and Logan's [42] work on witness-narratoragents, we created Town criers, in-game journalists who automatically pick up story events from anywhere in the world, and shout them in the city square. They also hold information about who carries the most evolved story artifact in the world.

6.1.3 Procedure. To prepare the game for official testing, we wanted the world to quickly appear populated and offer players clear, early goals to achieve. We scripted two custom events featuring blockades that sealed off the world and shrank the play space. One blockade to the main city could only be opened by defeating a difficult boss, while the other event set up several walls that blocked different paths, only breakable by a sufficiently skilled crafter player.

We announced the game's first official phase on the dedicated Discord channel, Facebook page and on the MMORPG subreddit ${ }^{5}$. We described all features of the game, but indicated that we primarily wanted feedback on story artifacts. As players started to join the game, we switched between playing with them, observing them, and fixing bugs. The game ran for 11 days, after which we asked four continuously active players to answer a questionnaire about how they liked story artifacts and town criers, and whether they felt that they had influenced the world.

\subsection{Results}

The We Ride technology probe demonstrated how to implement Narrative Substrates and provided first-impression feedback from players. They said they enjoyed interacting with player-generated histories, and generated numerous ideas for supporting persistence

\footnotetext{
${ }^{5}$ www.reddit.com/r/MMORPG
} 
in multiplayer game worlds. However, they also highlighted several design challenges, such as how to identify which events should remain relevant over long periods of time.

Players' Initial Thoughts. The four players who answered the questionnaire liked story artifacts: "[it] gives an element of story making" (P1) and "Holding a certain weapon that is known for its power [...] gives a unique feel to every character" (P4). They are "...really different from 'common items" (P3) because "you can develop some affection with a weapon that had been with you for lots of adventures" (P3). They felt that town criers: "make the world seem more alive and like actions matter" (P1) and "increase the feeling of immersion and make players feel like they had a true adventure" (P4). One player enjoyed seeing what other players had achieved, but wished that town criers "shared more notable achievements" (P2).

Challenges To Long-Term Relevance. Identifying events that remain relevant to players over longer periods of time is challenging. For example, we highlighted first-time experiences, and defined 'slaying a cave troll' as a story event, since these are relatively dangerous creatures. However, players became strong more quickly than expected: Once a player can repeatedly defeat a cave troll, it gets boring and only clutters up the story artifact's history description. Another limitation was the lack of accumulated past history. We had to generate each story artifact for the first time, which diminished player's ability to establish long-lasting influence.

\subsection{Deployment 2: Revisions and Extensions}

We incorporated our findings from deployment $D_{1}$, and developed new functionality to better support the rules of Narrative Substrates, with discoverability of old story artifacts. We also built an in-game interface for designers and GMs to generate new content by exposing and managing relationships among story events. Finally, we added location-centered story events to enable richer, more dynamic play and increase long-term relevance in stories.

Game World Changes. Over the course of a year, we completely overhauled the game, including two major changes: switching the camera view from $2.5 \mathrm{D}$ to $3 \mathrm{D}$, which provides greater visual depth and potential interaction techniques; and using UE4's built-in 'level streaming'-technique ${ }^{6}$, which significantly increases the size of the world and extends content, with only minor performance costs. The new version provided a more stable and scalable environment, with the potential for running sophisticated longitudinal studies.

6.3.1 Participants. 63 players played during deployment $D_{2}$; six provided continuous and detailed feedback on Discord.

6.3.2 Setup. We kept the story artifacts that were generated in $D_{1}$ and built support so they could be re-found as heirlooms. This marked the beginning of a new era and highlighted the fact that heirlooms are from a different time. When players first encounter an heirloom, its real name and story is concealed with a title of unsung, forgotten, mysterious, historical, chronicled, or ancient, to hint at how 'story rich' it is. To reveal the name and story, players must purchase or find a history book that corresponds to the correct title and look up the heirloom.

\footnotetext{
${ }^{6}$ Asynchronous loading of content based on distance at runtime.
}

We enabled GMs to access Narrative Substrates by building an in-game story monitor that let them search and filter events based on character, item and location and directly reify events on runtime (see Fig. 3). For this first implementation, GMs could specify an event and spawn an honor banner to acknowledge a player's past deeds (see Fig. 2). Acknowledged players can interact with the banner to temporarily increase their health, mana and hit rate. Other players can destroy the banner, but at the cost of becoming a criminal and reducing their karma. We also added basic functionality for GMs to directly spawn enemies that run out to attack players.

Deployment $D_{1}$ generated the idea of helping players use locations to create more dynamic stories. We wanted to simulate a sense of feeling 'at home' in friendly cities, and wariness after staying out in the wild too long. We named this concept astray and defined it as a variable between 0 and 1 that is multiplied by other factors in the environment. Each player character also continuously tracks how much 'impact' it has with the race of non-playing characters (NPC), such as orcs. If a player is seen by or slays an orc, their 'orc race impact' will increase by a base value that is then multiplied by astray. Depending on the current impact level, the system will spawn waves of NPCs of the same race, who will hunt and attack the player until their impact is zero again.

Impact is also connected to a 'raiding system'. If players accumulate a high impact level by consecutively defeating a race's dispatched hunters, the system will generate an 'outpost' which marks the player's last known location with a banner from where NPCs then continue to spawn. If the outpost is close to a friendly city and left undefeated, it triggers a raid. NPCs will gather and start to march on the city from two opposite directions. They will seize the city, unless players collaborate to defend it. If the city is besieged, friendly NPCs despawn and are replaced by the attackers. A city can only be reclaimed by defeating the race's leader.

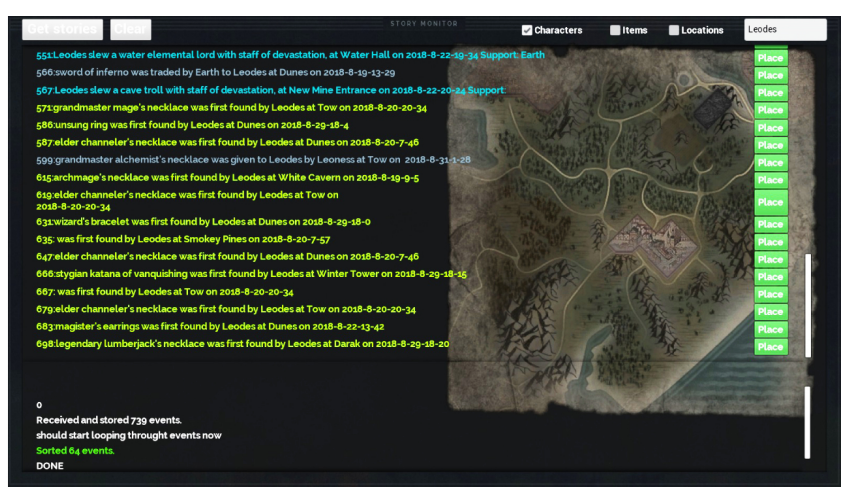

Figure 3. Story monitoring interface. Filtered story events with output log below (left). A worldmap (right) with buttons for placing an honor banner based on a story event. Searchbox and parameters for filtering (top).

6.3.3 Procedure. One week prior to deployment $D_{2}$, we posted a message via Discord, Reddit, and Facebook saying we planned to reset players' inventory and trained skills in a new 'season'. We said we would introduce a new game environment, test story features, and host organized GM-led events followed by feedback sessions. 
As players started to join the game, we switched between playing with them and fixing critical bugs they reported via Discord. We wanted to introduce players to heirlooms directly, so we added an NPC in the tutorial who describes a quest: "Look for an NPC in the first village you come to. If you complete the quest, you will receive an 'unsung' heirloom and a history book."

Nine days after the launch, we hosted a GM-led event created from players' activities and heirlooms from $D_{1}$. When we searched the Narrative Substrates for interesting story events, we found that a $D_{1}$ player had killed many witches. This became the plot of our main story: The Revenge of the Witches. In three scripted acts, we spawned NPCs who verbally referred to past events and placed related heirlooms on their corpses. Eleven days into deployment $D_{2}$, we asked players to give us feedback on our Discord channel, about what they liked and disliked about the event, the game, and how it could be improved.

\subsection{Results}

Deployment $D_{2}$ helped us assess the value of the Narrative Substrates concept, which: helps GMs directly reify stories and create events based on player histories; maintains the relevance of stories over time; and suggests new ways to reify meta persistent efforts, adding depth and meaning to their stories and game experiences.

GMs actively instrument Narrative Substrates. Designers and GMs take advantage of these powerful tools to filter and identify relevant story events, which in turn inspires new stories and the organization of narrative-driven events. For example, in $D_{2}$, we wrote a story that summarized and highlighted $D_{1}$ 's main characters and events. Players enjoyed this: "The overall storyline of the event was fun and made it easy to blame unexpected hiccups on the bad guys." (P2), even though they encountered several technical limitations, including lag and teleportation problems.

The process of searching and writing a script from the substrate led to new research questions: How can heirlooms that reference past narratives be associated with a specific main character's loot? How can we ensure that events are neither too easy nor too difficult? How can we spawn NPCs at runtime? Although we designed functionality for the last question, we dealt with the first two 'manually' during the $D_{2}$ event. We identified relevant heirlooms and relocated them to a GM's inventory by changing their IDs in the database. When a main character was defeated, the GM remained hidden and placed that character's heirlooms on the corpse. This functionality can be integrated directly into a story monitoring interface, with additional techniques for handling links and relationships, to improve how Narrative Substrates adapt to characters, heirlooms, and their narratives.

Narrative Substrates Support Long-term Story Relevance. We addressed the challenge of long-term relevance by introducing story artifacts generated by players in $D_{1}$. Although this did not directly address the issue of repetitive cave troll stories in deployment $D_{1}$, it offered players a new perspective, where history is present as an active element of the game: "This unsung item I mean, checking the history of a weap[on] is a feature that I've never seen before" (P1). Implementing heirlooms also revealed a flaw in $D_{1}$ : if a story artifact decays or is destroyed, all of its narrative content is permanently erased. This crucial but hidden problem led to a new insight: story events should always persist in the database, even if not currently visible in the game.

We also found that exploring location-based story events with astray, impact and raids revealed further need of functionality to let designers and GMs set and tune the rates at which different story events and artifacts grow and decay, to help sort out relevance issues. e.g. an honor banner's effect can decay with time.

Narrative Substrates Reveal Ideas to Reify Meta Persistence. One direct benefit of our approach is the close relationship we established with the game's player community. Since deployment $D_{1}$, a number of players have expended extraordinary efforts to explore every aspect of the game. They voluntarily provided us with multiple examples of meta persistence, including spreadsheets and graphs that track output values such as skill gain and damage; wikis and walk-through videos; discussions surrounding changes; and comparative analysis of We Ride with similar games. Their examples prompted ideas for extending Narrative Substrates by reifying players' research activities, e.g. creating in-game wikis for players to submit information, or offering unique items to players to acknowledge their bug reports.

After announcing deployment $D_{2}$, the Unreal Engine 4 developer community recognized We Ride. One person wrote on Discord: "Awsome. Really like your conceptualization of persistence. So lacking in modern games, not just mmo's" (P6). We were featured in UE4 community spotlight ${ }^{7}$, a weekly Youtube series that highlights developers who use Unreal Engine. They also provided a link to the game on the front page of the Epic Games Launcher. This level of interest is encouraging feedback about the benefits of using Narrative Substrates to create persistent game worlds.

\section{CONCLUSION AND FUTURE WORK}

This paper explores the role of persistence in increasing player influence in persistent game worlds. We reify the narratives that emerge from players' actions into Story Artifacts, which are directly represented and interacted with in play.

Our first two empirical studies identified two main themes with respect to players' experiences: uniqueness and first-time experiences and meta persistence. Study three tested these themes and also found that: lack of world persistence reduces uniqueness and meta persistence significantly affects gameplay. We replicated these findings in study four and gained further insights about players' first-time experiences, and how persistence affects their ability to discover and explore game worlds. These results, combined with earlier work on persistence, narrative theory and instrumental interaction, led us to develop Narrative Substrates, a theoretical framework for designing game infrastructures that reify and manage emergent narratives in persistent game worlds. Game designers, game masters and players can repurpose persisted traces of player activity into unique, interactive content. We developed We Ride, a live MMORPG, using the principles of Narrative Substrates, and deployed it as a two-phase technology probe over a period of a year. This provided a continuous stream of feedback from the player community, and revealed

\footnotetext{
${ }^{7}$ Community spotlight: https://youtu.be/TygjPe9XHTw?t=433
} 
both the benefits and challenges of the approach, as well as generating implications for design. Narrative Substrates clearly shows the benefits of persisting traces of player activity, without generating conflicts between players' individual experiences, designers' control of the world, and overarching narratives.

Currently, We Ride simply demonstrates some of the potential for a Narrative Substrates approach to building MMORPGs. Future work will investigate how capturing player's activities and representing them in the world leads towards our long-term goal of making players truly feel like legends who are remembered and influential in the game world and beyond, while supplying designers with an almost infinite stream of meaningful new content.

\section{ACKNOWLEDGMENTS}

This project has received funding from the European Research Council (ERC) under the European Union's Horizon 2020 research and innovation programme (grant agreement № № 321135 “CREATIV: Creating Co-Adaptive Human-Computer Partnerships”)”.

\section{REFERENCES}

[1] ArenaNet. 2012. Guild Wars 2. Game [Microsoft Windows]. NCSOFT Corporation, Pangyo, Seongnam, South Korea.

[2] Richard Bartle. 1999. Multi-User Dungeons. Retrieved June 30, 2019 from https: //mud.co.uk/richard/ifan294.htm

[3] R Bartle. 2016. MMOs from the inside out. Springer

[4] Richard A Bartle. 2003. Designing virtual worlds. New Riders.

[5] Richard A Bartle. 2016. The decline of MMOs. In Global Game Industries and Cultural Policy. Springer, 303-316.

[6] Michel Beaudouin-Lafon. 2000. Instrumental Interaction: An Interaction Model for Designing post-WIMP User Interfaces (CHI '00). ACM, New York, NY, USA, 446-453. https://doi.org/10.1145/332040.332473

[7] Michel Beaudouin-Lafon. 2004. Designing Interaction, Not Interfaces (AVI '04) ACM, New York, NY, USA, 15-22. https://doi.org/10.1145/989863.989865

[8] Michel Beaudouin-Lafon. 2017. Towards Unified Principles of Interaction (CHItaly '17). ACM, New York, NY, USA, Article 1, 2 pages. https://doi.org/10.1145/3125571. 3125602

[9] Michel Beaudouin-Lafon and Wendy E. Mackay. 2000. Reification, Polymorphism and Reuse: Three Principles for Designing Visual Interfaces (AVI '00). ACM, New York, NY, USA, 102-109. https://doi.org/10.1145/345513.345267

[10] Blizzard Entertainment. 2005. World of Warcraft. Game [Microsoft Windows]. Blizzard Entertainment, Texas, U.S.

[11] Virginia Braun and Victoria Clarke. 2006. Using thematic analysis in psychology. Qualitative research in psychology 3, 2 (2006), 77-101.

[12] Marcus Carter, Martin Gibbs, and Mitchell Harrop. 2012. Metagames, Paragames and Orthogames: A New Vocabulary (FDG '12). ACM, New York, NY, USA, 11-17. https://doi.org/10.1145/2282338.2282346

[13] CCP Games. 2003. EVE Online. Game [Microsoft Windows]. Simon \& Schuster, Inc, New York, U.S.

[14] Marianela Ciolfi Felice, Nolwenn Maudet, Wendy E. Mackay, and Michel Beaudouin-Lafon. 2016. Beyond Snapping: Persistent, Tweakable Alignment and Distribution with StickyLines (UIST '16). ACM, New York, NY, USA, 133-144. https://doi.org/10.1145/2984511.2984577

[15] Suzanne de Castell, Nicholas Taylor, Jennifer Jenson, and Mark Weiler. 2012. Theoretical and Methodological Challenges (and Opportunities) in Virtual Worlds Research (FDG '12). ACM, New York, NY, USA, 134-140. https://doi.org/10.1145/ 2282338.2282366

[16] M. De Plater. 2015. Postmortem: Monolith Productions' Middle-earth: Shadow of Mordor. Retrieved July 12, 2018 from https:/www.gamasutra.com/view/news/ 234421/Postmortem_Monolith_Productions_Middleearth_Shadow_of_Mordor php

[17] Thomas Debeauvais, Cristina V Lopes, Nick Yee, and Nicolas Ducheneaut. 2014 Retention and progression: Seven months in World of Warcraft.. In FDG.

[18] A. Dieberger, P. Dourish, K. Höök, P. Resnick, and A. Wexelblat. 2000. Social Navigation: Techniques for Building More Usable Systems. interactions 7, 6 (Nov. 2000), 36-45. https://doi.org/10.1145/352580.352587

[19] Paul Dourish and Matthew Chalmers. 1994. Running out of space: Models of information navigation. In Short paper presented at HCI'94, Vol. 94. 23-26.

[20] Mirjam Eladhari and Craig Lindley. 2004. Story construction and expressive agents in virtual game worlds. In Proceedings of the Other Players Conference, Copenhagen, Denmark. 63-71.
[21] Mirjam Palosaari Eladhari. 2018. Re-Tellings: The Fourth Layer of Narrative as an Instrument for Critique. In Interactive Storytelling, Rebecca Rouse, Hartmut Koenitz, and Mads Haahr (Eds.). Springer International Publishing, Cham, 65-78.

[22] Clive Fencott. 2001. Virtual storytelling as narrative potential: Towards an ecology of narrative. In International Conference on Virtual Storytelling. Springer, 90-99.

[23] John C Flanagan. 1954. The critical incident technique. Psychological bulletin 51, 4 (1954), 327.

[24] B. Francis. 2017. Upgrading the Nemesis system for Middle-earth: Shadow of War. Retrieved July 23, 2018 from https://www.gamasutra.com/view/news/305601/ Upgrading_the_Nemesis_system_for_Middleearth_Shadow_of_War.php

[25] FromSoftware. 2015. Bloodborne. Game [PlayStation 2]. Sony Computer Entertainment, Tokyo, Japan.

[26] Frontier Developments. 2005. Elite Dangerous. Game [Microsoft Windows]. Froniter Developments, Cambridge, England.

[27] Jérémie Garcia, Theophanis Tsandilas, Carlos Agon, and Wendy E. Mackay. 2014. Structured Observation with Polyphony: A Multifaceted Tool for Studying Music Composition (DIS '14). ACM, New York, NY, USA, 199-208. https://doi.org/10. $1145 / 2598510.2598512$

[28] Carla Griggio. 2018. Designing for Ecosystems of Communication Apps. Theses. Université Paris-Saclay. https://tel.archives-ouvertes.fr/tel-02157268

[29] William C. Hill, James D. Hollan, Dave Wroblewski, and Tim McCandless. 1992. Edit Wear and Read Wear (CHI '92). ACM, New York, NY, USA, 3-9. https: //doi.org/10.1145/142750.142751

[30] James Hollan, Edwin Hutchins, and David Kirsh. 2000. Distributed cognition: toward a new foundation for human-computer interaction research. ACM Transactions on Computer-Human Interaction (TOCHI) 7, 2 (2000), 174-196.

[31] Hilary Hutchinson, Wendy Mackay, Bo Westerlund, Benjamin B. Bederson, Allison Druin, Catherine Plaisant, Michel Beaudouin-Lafon, Stéphane Conversy, Helen Evans, Heiko Hansen, Nicolas Roussel, and Björn Eiderbäck. 2003. Technology Probes: Inspiring Design for and with Families (CHI '03). ACM, New York, NY, USA, 17-24. https://doi.org/10.1145/642611.642616

[32] IGN.com. 2018. Nemesis System. Retrieved July 14, 2018 from http://www.ign. com/wikis/shadow-of-war/Nemesis_System

[33] Clemens N. Klokmose, James R. Eagan, Siemen Baader, Wendy Mackay, and Michel Beaudouin-Lafon. 2015. Webstrates: Shareable Dynamic Media (UIST '15). ACM, New York, NY, USA, 280-290. https://doi.org/10.1145/2807442.2807446

[34] Raph Koster. 2008. Red 5s chasing the persistence dream. https://www.raphkoster. com/2008/08/25/red-5s-chasing-the-persistence-dream/

[35] Raph Koster. 2009. Defining persistence for MMOs. https://www.raphkoster.com/ 2009/06/01/defining-persistence-for-mmos/

[36] Ralph Koster. 2009. A Sandbox to Play In. https://www.raphkoster.com/games/ insubstantial-pageants/a-sandbox-to-play-in/

[37] Max Kreminski, Ben Samuel, Edward Melcer, and Noah Wardrip-Fruin. 2019. Evaluating AI-Based Games Through Retellings. In Proceedings of the Fifteenth AAAI Conference on Artificial Intelligence and Interactive Digital Entertainment (AIIDE-19). AAAI.

[38] JC Kroon. 2016. Nemesis Narratives: The relationship between embedded and emergent narrative in Middle Earth: Shadow of Mordor. Master's thesis.

[39] Wendy Mackay. 2002. Using video to support interaction design. DVD Tutorial. INRIA and ACM/SIGCHI. 88 pages.

[40] Wendy Mackay. 2019. Designing with Sticky Notes. In Sticky Creativity: Post-It Note Cognition, Interaction and Digitalization, Halskov Christensen and Clemens Klokmose (Eds.). Academic Press. In print.

[41] Wendy E Mackay and Anne-Laure Fayard. 1997. HCI, natural science and design: a framework for triangulation across disciplines. In Symposium on Designing Interactive Systems: Proceedings of the 2 nd conference on Designing interactive systems: processes, practices, methods, and techniques, Vol. 18. 223-234.

[42] Neil Madden and Brian Logan. 2007. Collaborative Narrative Generation in Persistent Virtual Environments.. In AAAI Fall Symposium: Intelligent Narrative Technologies. 71-78.

[43] Monolith Productions. 2005. The Matrix Online. Game [Microsoft Windows]. SEGA, Tokyo, Japan. Warner Bros. Interactive Entertainment, California, U.S.

[44] Monolith Productions. 2017. Middle-earth: Shadow of War. Game [Microsoft Windows]. Warner Bros. Interactive Entertainment, California, U.S.

[45] Origin Systems. 1997. Ultima Online. Game [Microsoft Windows]. Electronic Arts, Texas, U.S.

[46] James Pita, Brian Magerko, and Scott Brodie. 2007. True Story: Dynamically Generated, Contextually Linked Quests in Persistent Systems (Future Play '07). ACM, New York, NY, USA, 145-151. https://doi.org/10.1145/1328202.1328228

[47] Reddit. 2019. Gamers of Reddit, what gaming experience will you never forget and why? Retrieved February 1, 2020 from https://www.reddit.com/r/AskReddit/ comments/bgko0b/gamers_of_reddit_what_gaming_experience_will_you/

[48] Reddit. 2019. What games foster players to create their own legends? Retrieved January 31, 2020 from https://www.reddit.com/r/truegaming/comments/8vjvgr/ what games foster players to create their own/

[49] James Ryan. 2018. Curating Simulated Storyworlds. Ph.D. Dissertation. UC Santa Cruz. 
[50] Katie Salen, Katie Salen Tekinbaş, and Eric Zimmerman. 2004. Rules of play: Game design fundamentals. MIT press.

[51] Ansel Arjan Schütte. 1998. Patina: layering a history-of-use on digital objects. Master's thesis. Massachusetts Institute of Technology.

[52] Square Enix. 2013. Final Fantasy XIV. Game [Microsoft Windows]. Square Enix, Tokyo, Japan.

[53] Anders Tychsen and Michael Hitchens. 2006. Ghost worlds-time and consequence in MMORPGs. In International Conference on Technologies for Interactive Digital Storytelling and Entertainment. Springer, 300-311.

[54] Anders Tychsen, Michael Hitchens, Thea Brolund, and Manolya Kavakli. 2005 The game master. In Proceedings of the second Australasian conference on Interactive entertainment. Creativity \& Cognition Studios Press, 215-222.
[55] We Ride. 2018. We Ride. Game [Microsoft Windows]. http://www.weridegame. com We Ride, Stockholm, Sweden.

[56] Alan Wexelblat and Pattie Maes. 1999. Footprints: History-rich Tools for Information Foraging (CHI '99). ACM, New York, NY, USA, 270-277. https: //doi.org/10.1145/302979.303060

[57] Bloodborne Wiki. 2019. Notes. Retrieved March 22, 2019 from http://bloodborne. wikia.com/wiki/Notes

[58] Nick Yee. 2014. The Proteus paradox: How online games and virtual worlds change us-and how they don't. Yale University Press.

[59] Greg Zeschuk. 2011. DICE Keynote Panel. 